\title{
Medicina y Seguridad del Trabajo, evaluación desde su ingreso en la Red SciELO (ScientificElectronic Library Online)
}

\section{Medicina y Seguridad del Trabajo, evaluation since joining SciELO (Scientific Electronic Library Online)}

\author{
Ascensión Bernal Zamora ', Jerónimo Maqueda Blasco 2, Jorge Veiga de Cabo ${ }^{3}$, Marcelo D’Agostino 4 \\ 1. Directora. Medicina y Seguridad del Trabajo. Instituto de Salud Carlos III. Madrid. España. \\ 2. Director Adjunto. Medicina y Seguridad del Trabajo. Instituto de Salud Carlos III. Madrid. España. \\ 3. Redactor Jefe. Medicina y Seguridad del Trabajo. Instituto de Salud Carlos III. Madrid. España. \\ 4. Redactor Adjunto. Medicina y Seguridad del Trabajo. Organización Panamericana de la Salud, Oficina Regional \\ para las Américas de la Organización Mundial de la Salud (OPS/OMS). Washington DC. EUA.
}

Resumen

Se realiza una revisión de los principales cambios editoriales y de contenido sufridos por la revista de Medicina y Seguridad del Trabajo durante los últimos 5 años, coincidiendo con el periodo desde que ingresó en SciELO (ScientificElectronic Library Online) (2007-2011). En el periodo estudiado se han realizado un número de consultas a sus artículos superior a los 190 mil, ha tenido un Factor de Impacto (FI) SciELO del 1.22, se ha incrementado el número de artículos recibidos de diferentes países de América y Europa y se la revista ha sido indizada en diferentes Bases de Datos bibliográficas internacionales (SciELO, Directory of Open Access Journals (DOAJ), Google Schoolar, Eprints in Library and InformationScience (E-LIS) $y$ LATINDEX) y nacionales (IBECS, IME, Base de Datos del Consejo Superior de Investigaciones Científicas (CSIC), DIALNET y CUIDEN). La calidad editorial y de contenidos también se han visto mejoradas con diferentes modificaciones y cambios introducidos. Se cuenta con la participación editorial de la Organización Panamericana de la Salud, Oficina regional para las Américas de la Organización Mundial del Salud (OPS/ OMS), se ha producido un incremento superior al $40 \%$ de los artículos publicados en el último quinquenio respecto al periodo anterior, pasando de 118 a 202 artículos. El número de Editoriales y casos Clínicos también se incrementan en un porcentaje del $40 \%$. Los Artículos Originales se incrementan en algo más del 50\% (53 versus 109), encontrando el mayor incremento en los Artículos de revisión que pasan de 3 en el primer anterior a 25 en el periodo de estudio. Por último, en 2011, Medicina y Seguridad en el Trabajo ha publicado por primera vez un número monográfico especial sobre Factores Psicosociales, Salud laboral y Salud Mental que incluye 15 artículos de revisión sobre temas de actualidad realizados por especialistas en la materia. Los resultados estimulan el afán por del Equipo Editorial por seguir trabajando para mejorar de calidad y visibilidad de la revista durante los próximos años.

Med Segur Trab (Internet) 2012; 58 (226) 1-5

Palabras Clave: Salud Laboral, Salud Ocupacional, Medicina del Trabajo, Edición, Publicación, Acceso Abierto, SciELO.

Abstract

This Editorial is a review of key content and editorial changes suffered by the Medicina $y$ SeguridaddelTrabajo for the past 5 years, coinciding with the period since joining SciELO (Scientific Electronic Library Online) (2007-2011). In the study period there have been more of 190,000 articles access, an impact factor (IF) SciELO of 1.22, increase the number of articles received from different countries of America and Europe and the journal has been indexed in various international bibliographic databases 
(SciELO, Directory of Open Access Journals (DOAJ), Google Schoolar, Eprints in Library and Information Science (E-LIS) and LATINDEX) and national (IBECS, EMI, Base Data National Research Council (CSIC), and CUIDEN DIALNET). Editorial quality and contents have also been enhanced with various modifications and changes, and the Pan American Health Organization, Regional Office for the Americas of the World Health Organization (PAHO/WHO)is involved in the journal edition. There was an increase of over $40 \%$ of articles published in the last five years compared to previous period, from 118 to 202 items. The number of Editorials and Clinical cases are also increasing at a rate of $40 \%$. Original Articles are increased by just over $50 \%$ ( 53 versus 109), finding the largest increase in Review articles ( 3 in the first period and 25 in the last five-years study period). Finally, in 2011, Medicina y SeguridaddelTrabajo has first published a special number about Psychosocial Factors, and Mental Health Occupational Health including 15 review articles on current topics by specialists in the field. This results, encourage Editorial Team eagerness to continue working to improve quality and visibility of the journal over the coming years.

Med Segur Trab (Internet) 2012; 58 (226) 1-5

Key Words: Labor Health, Occupational Health, Medicine, Work, Edition, Publication, Open Access, SciELO. 


\section{IMPULSO QUE HA SUPUESTO PARA DE LA REVISTA MEDICINA Y SEGURIDAD DEL TRABAJO SU ENTRADA EN LA RED SCIELO}

En 2011 se cumplieron 5 años desde que en 2007, la revista de Medicina y Seguridad del Trabajo ingresó en la Red SciELO (ScientificElectronic Library Online), con lo que se cumplía una de las primeras estrategias encaminadas a incrementar su visibilidad a nivel nacional e internacional. ${ }^{1}$

De esta forma, teniendo en cuenta sólo a los accesos a la revista registrados a través del Portal SciELO durante este periodo, la cifra supera los 360 mil accesos a diferentes artículos publicados en la misma y con un Factor de Impacto de 1.22 en dos últimos años (http://scielo.isciii.es, consultado abril 2012), lo que sin lugar a dudas ha repercutido de forma importante sobre el incremento de su visibilidad, no sólo por lo que supone esta cifra de accesos, sino porque además en este mismo periodo se ha notado un incremento importante del número de artículos recibidos procedentes de otros países como Bélgica, Italia y Portugal,en Europa, y deArgentina, Brasil, Bolivia, Chile, Colombia, Cuba, Ecuador, EEUU, México y Perú, procedentes del Continente Americano, se ha visto bastante incrementada respecto al periodo anterior a su ingreso en la Red SciELO.

Otra dato importante a tener en cuenta en este mismo sentido, es que, a fecha de la publicación de este Editorial, el Portal SciELO-España ocupa el octavo puesto en el Ranking Web de Repositorios del Mundo que elabora el Laboratorio de Cibermetría del Consejo Superior de Investigaciones Científicas (CSIC) de España ${ }^{2}$, oscilando sus posiciones en los últimos 5 meses entre el séptimo y el decimoprimero.

Tampoco debemos olvidar que la incorporación de Medicina y Seguridad en el Trabajo a la Red SciELO ha facilitado su aparición en el Directory of Open Access Journals (DOAJ) y en Google Schoolar, mejorando su visibilidad en Bases de datos Internacionales como Eprints in Library and InformationScience (E-LIS) $y$ LATINDEX, así como en las bases de datos bibliográficas nacionales, entre las que podemos destacar el Índice Bibliográfico Español en Ciencias de la Salud (IBECS), Índice Médico Español (IME), Base de Datos del Consejo Superior de Investigaciones Científicas (CSIC), DIALNET y CUIDEN. ${ }^{3-6}$

Durante este tiempo, otro de nuestros objetivos y principales compromisos ha sido intentar mejorar la calidad de la publicación, tanto desde su vertiente editorial como de calidad de contenidos.

\section{MEDIDAS ADOPTADAS PARA MEJORAR LA CALIDAD EDITORIAL}

En cuanto al compromiso de mejorar la calidad editorial, se ha procurado tener un mayor rigor en el cumplimiento de normas nacionales e internacionales en lo que respecta a presentación formal de formatos de revistas científicas, introduciendo mejoras relacionadas con la identidad de la revista como la incorporación de la Institución editora de la publicación y el logo en portada. A partir de 2006 se solicita anualmente y se incorpora el Número de Identificación de Publicaciones Oficiales (NIPO)y como a partir de 2009 la revista se edita exclusivamente en formato electrónico, se ha sustituido su antiguo ISSN de la extinguida versión en papel (0465-546X) por el asignado a su nueva versión en formato de la edición exclusivamente electrónica(1989-7790).

Otra de las modificaciones que se han introducido, y que afecta mejorando la identidad de la revista, es que a partir del último número de diciembre de 2008 aparece tanto en portada como en cada una de sus páginas, el periodo de meses a los que corresponde el número. También siguiendo Normas ISO, ${ }^{7}$ se cambia la numeración romana que se venía utilizando hasta entonces para identificar los números correspondientes a cada tomo por la numeración arábica, además de sustituir la paginación de los artículos de forma individual para cada número, como se venía haciendo, por la paginación continua para todos los artículos de un mismo tomo, lo que permite una mejor identificación de los contenidos por años como es habitual encontrar en la gran mayoría de las revistas científicas.

Para finalizar este apartado relativo a las modificaciones y mejoras relacionadas con la calidad editorial, en estos últimos años se ha realizado un esfuerzo por homogeneizar el formato de los artículos en lo que respecta a estructura y maquetado de texto, tablas y figuras. Los títulos, resúmenes y palabras clave de todos los artículos y casos clínicos, se publican en español e inglés, lo que facilita y permite incrementar su visibilidad y difusión internacional. Se ha puesto especial cuidado en establecer un sistema de normalización para los criterios de filiación de los autores e instituciones a las que pertenecen, indicando además el país 
de las instituciones, dado que hay ciudades en España y Latinoamérica que tienen nombres iguales. También, siempre que se puede, se ha incluido la dirección del autor responsable derecibir la correspondencia correspondiente a cada uno de los artículos que se publican, la fecha de aceptación del mismo y la identificación del número, tomo y la paginación de cada de ellos, datos que figuran en la parte superior de cada una de las páginas de todos los artículos.

\section{MEDIDAS ADOPTADAS PARA MEJORAR LA CALIDAD DE CONTENIDOS}

En cuanto la las variaciones que se han introducido intentando mejorar la calidad de contenidos, en primer lugar, se han elaborado unas Normas de Publicación más amplias y detalladas que las existentes anteriormente a su incorporación en SciELO en 2007. ${ }^{8}$

Se ha establecido un único Comité de Editorial con un equipo responsablecon participación de la Organización Panamericana de la Salud, Oficina Regional para las Américas de la Organización Mundial de la Salud (OPS/OMS) y en el que también se ha intentado diversificar la participación, tanto de sus miembros como los del Comité Científico, en cuanto a las instituciones nacionales e internacionales que representan. ${ }^{?}$

Si comparamos la producción científica de la revista durante el periodo que estamos evaluando desde su inclusión en la Red SciELO (periodo 2007-2011) respecto al quinquenio inmediatamente anterior (2002-2006), observamos que cuantitativamente se ha producido un incremento superior al $40 \%$ de los artículospublicados entre ambos quinquenios en sus diferentes modalidades, pasando de 118 artículos publicados en el periodo 2002-2006 a 202 artículos en el periodo (eliminar 3)2007-2011 (Tablas I y II), aunque debemos tener en cuenta que durante 2002 y 2003 la publicación estuvo prácticamente interrumpida y sólo se publicó un número de la revista en 2003

Tabla I. Distribución de artículos publicados por años y tipología. Periodo 2002-2006.

\begin{tabular}{|c|c|c|c|c|c|c|c|c|c|c|}
\hline Año & Editoriales & $\begin{array}{l}\text { Artículos } \\
\text { opinión }\end{array}$ & $\begin{array}{l}\text { Artículos } \\
\text { originales }\end{array}$ & $\begin{array}{l}\text { Artículos } \\
\text { revisión }\end{array}$ & $\begin{array}{c}\text { Artículos } \\
\text { actualización }\end{array}$ & $\begin{array}{l}\text { Casos } \\
\text { clínicos }\end{array}$ & Legislación & Congresos & $\begin{array}{c}\text { In } \\
\text { memoriam }\end{array}$ & TOTAL \\
\hline 2006 & 4 & 3 & 14 & 2 & 3 & 1 & 5 & - & - & 32 \\
\hline 2005 & 4 & 4 & 14 & - & 5 & 1 & 5 & 1 & 1 & 35 \\
\hline 2004 & 4 & 4 & 18 & 1 & 5 & 2 & 8 & - & - & 42 \\
\hline 2003 & - & - & 7 & - & - & - & 2 & - & - & 9 \\
\hline 2002 & - & - & - & - & - & - & - & - & - & - \\
\hline TOTAL & 12 & 11 & 53 & 3 & 13 & 4 & 20 & $\mathbf{1}$ & $\mathbf{1}$ & 118 \\
\hline
\end{tabular}

Tabla II. Distribución de artículos publicados por años y tipología. Periodo 2007-2011.

\begin{tabular}{|c|c|c|c|c|c|c|c|c|c|c|c|}
\hline Año & Editoriales & $\begin{array}{l}\text { Artículos } \\
\text { especiales }\end{array}$ & $\begin{array}{l}\text { Artículos } \\
\text { originales }\end{array}$ & $\begin{array}{l}\text { Artículos } \\
\text { revisión }\end{array}$ & Retrospectivas & Prospectivas & $\begin{array}{c}\text { Aula } \\
\text { abierta }\end{array}$ & $\begin{array}{l}\text { Casos } \\
\text { clínicos }\end{array}$ & $\begin{array}{l}\text { Cartas } \\
\text { director }\end{array}$ & $\begin{array}{c}\text { Comentario } \\
\text { libros }\end{array}$ & TOTAL \\
\hline 2011 & 5 & 5 & 26 & 15 & - & - & - & 1 & 1 & - & 53 \\
\hline 2010 & 4 & 2 & 16 & 3 & 1 & - & 1 & 3 & - & - & 30 \\
\hline 2009 & 4 & 3 & 25 & 3 & 1 & - & - & 1 & - & - & 37 \\
\hline 2008 & 4 & 8 & 26 & - & 1 & - & 4 & 1 & 1 & 1 & 46 \\
\hline 2007 & 4 & 6 & 16 & 4 & 1 & 1 & - & - & - & 4 & 36 \\
\hline TOTAL & 21 & 24 & 109 & 25 & 4 & 1 & 5 & 6 & 2 & 5 & 202 \\
\hline
\end{tabular}

Otras medidas que se han introducido en este último periodo, todas ellas tendentes a mejorar la calidad de contenidos, han sido la sustitución de Artículos de Opinión por Artículos Especiales, ya que los primeros admiten visiones más subjetivas mientras que los segundos permiten una orientación más objetiva basada en el análisis de determinados temas realizado por expertos.

Se crean también tres secciones nuevas, Retrospectivas, Perspectivas y Aula Abierta, eliminando las secciones de Legislación y Congresos que se consideraban de escaso interés científico para la revista. También 
se crea la sección de Cartas la director que le confiere un carácter de apertura hacia la comunicad científica y facilita la interacción de masa crítica.

Otros datos de interés que encontramos cuando comparamos ambos periodos es que el número de Editoriales y casos Clínicos también se incrementan en un porcentaje del 40 \%. Los Artículos Originales se incrementan en algo más del 50\% (53 respecto a 109), encontrando el mayor incremento en los Artículos de revisión que pasan de 3 en el primer periodo a 25 en el segundo.

Además de estos cambios, en los últimos años, la revista viene publicando en el último número de cada año un índice de artículos y otro de autores, así como la relación del os evaluadores que han intervenido en la revisión de manuscritos para ser publicados por la revista.

Por último, en 2011, Medicina y Seguridad en el Trabajo ha publicado por primera vez un número monográfico especial sobre Factores Psicosociales, Salud laboral y Salud Mental que incluye 15 artículos de revisión sobre temas de actualidad realizados por especialistas en la materia.

Los resultados del análisis sobre este último periodo, estimulan nuestro interés y afán por seguir trabajando para mejorar de calidad y visibilidad de la revista durante los próximos años. Queremos agradecer a todos los miembros, tanto del Comité Editorial como del Científico, sus aportaciones y su participación activa para alcanzar dichas mejoras, a los autores que con su esfuerzo, trabajos y envío de sus artículos han contribuido de forma tan importante a la misma y naturalmente, a todos los lectores y lectoras, que además de constituir el objeto principal de la publicación, con su fidelidad permiten mantener la continuidad de la revista.

\section{BIBLIOGRAFÍA}

1. Veiga-Cabo J. Medicina y seguridad del trabajo ha superado los criterios de selección para integrarse en la Red Scielo (ScientificElectronic Library On Line) Med. segur. Trab. 2007;53(207):1-4. (Consultado 16 de febrero de 2012). Disponible en: http://scielo.isciii.es/scielo.php?script=sci_arttext\&pid=S0465-546X2007000200002\&lng=es; http://dx.doi.org/10.4321/S0465$546 \times 2007000200002$

2. Ranking Web de Repositorios del Mundo que elabora el Laboratorio de Cibermetría del Consejo Superior de Investigaciones Científicas (CSIC) de España. (Consultado: 1 de marzo de 2012). Disponible en: http://repositories.webometrics.info/topportals_ es.asp

3. Veiga de Cabo J, Martín-Pastor B, Calvo-Tello M, Pablos-Martín M. El modelo SciELO y su contribución a la difusión de las revistas de ciencias de la salud españolas. RCOE. 2003;8(1):67-72. (Consultado: 16 de febrero 2012). Disponible en: http://scielo. isciii.es/scielo.php?script=sci_arttext\&pid=S1138-123X2003000100005\&lng=es\&nrm=iso; http://dx.doi.org/10.4321/S1138123X2003000100005.

4. Veiga de Cabo, Jorge. ScientificElectronic Library Online (SciELO) y Oncología. Oncología (Barc.), Set 2004, vol.27, no.9, p.13-18. (Consultado: 16 de febrero 2012).Disponible en: http://scielo.isciii.es/scielo.php?script=sci_arttext\&pid=S037848352004000900001\&lng=es\&nrm=iso http://dx.doi.org/10.4321/S0378-48352004000900001

5. Veiga de Cabo J. Visibilidad de revistas científicas e iniciativas para incrementar la difusión de las publicaciones españolas. Nutr. Hosp. 2003; 18(4): 177-180. (Consultado: 16 de febrero de 2012). (Consultado: 16 de febrero de 2012). Disponible en: http://scielo. isciii.es/scielo.php?script=sci_arttext\&pid=S0212-16112003000400001\&lng=es

6. Sanz-Valero Javier, D’Agostino Marcelo José, Castiel Luis David, Veiga de Cabo Jorge. La iniciativa Open Access, una visión de conjunto. Med. segur. trab. 2007;53(207): 05-09. (Consultado: 16 de febrero de 2012). Disponible en: http://scielo.isciii.es/scielo. php?script=sci_arttext\&pid=S0465-546X2007000200003\&lng=es; http://dx.doi.org/10.4321/S0465-546X2007000200003

7. Normas ISO de presentación de publicaciones periódicas científicas. Boletín de la ANABAD 1997;XLVII(2):169-192.

8. Sanz-Valero, J; Wanden-Berghe, C; García de Lorenzo, A; Gil, A; Pérez de la Cruz, A; Sánchez-Álvarez, C; Veiga-Cabo, J; Laborda, 1; Culebras, J. Las normas de publicación de Nutrición Hospitalaria; un futuro para la revista en beneficio de sus autores. Nutr. Hosp. 2007;22(1):1-3. (Consultado: 16 de febrero de 2012). Disponible en: http://scielo.isciii.es/scielo.php?script=sci_ arttext\&pid=S0212-16112007000100001\&lng=es

9. Veiga de Cabo Jorge, Maqueda Blasco Jerónimo, Andrus Jon, Gross Socorro, CassanhaGalvaoLuiz A, Corvalan Carlos Florencio et al . Marco de cooperación entre la Organización Panamericana de la Salud (OPS), Oficina Regional para las Américas de la Organización Mundial de la Salud (OMS) y la Escuela Nacional de Medicina del Trabajo del Instituto de Salud Carlos III en materia de Salud y Seguridad en el Trabajo. Med. segur. trab. [Internet]. 2010;56(221):266-270. (Consultado 16 de febrero de 2012). Disponible en: http://scielo.isciii.es/scielo.php?script=sci_arttext\&pid=S0465-546X2010000400001\&lng=es; http://dx.doi. org/10.4321/S0465-546X2010000400001 